\title{
Short-Term Clinical Outcomes and Comparison of Ultrasound Versus Magnetic Resonance Imaging of Superior Capsular Reconstruction
}

\author{
Brian L. Badman, M.D., Aaron M. Baessler, M.D., and Molly Moor, Ph.D., M.P.H.
}

\begin{abstract}
Purpose: To evaluate the short-term outcomes of 10 patients with irreparable massive rotator cuff tears treated with arthroscopic superior capsular reconstruction (SCR) using dermal allograft. Methods: Between 2016 and 2018, patients with symptomatic irreparable rotator cuff tears were prospectively enrolled for treatment with arthroscopic SCR. Investigational review board approval was achieved. All patients were treated by a single fellowship-trained shoulder surgeon. Minimum follow-up was 1 year. Range of motion and functional outcome according to visual analog scale pain, American Shoulder and Elbow Surgeons, and Simple Shoulder Test scores were assessed preoperatively and at routine follow-up intervals. Magnetic resonance imaging (MRI) and ultrasound were obtained at a minimum of 1 year to assess graft integrity and to correlate clinical outcomes. Results: Ten patients with a mean age of 58.6 years had a minimum follow-up of 1 year. In all patients, preoperatively to postoperatively, mean forward flexion improved from $141^{\circ}$ to $173^{\circ}(P=.018)$, mean visual analog scale pain score decreased from 6.5 to $1(P=.004)$, and mean American Shoulder and Elbow Surgeons score improved from 43 to $87(P=.005)$. At 1 year, ultrasound evaluation identified graft failure in 1 patient $(10 \%)$, whereas MRI diagnosed graft failure in 7 patients $(70 \%)$. Of the 7 failures diagnosed by MRI, 4 failed at the level of the glenoid, 2 failed mid-graft, and 1 failed at the humerus. Conclusions: Although clinical outcomes are statistically improved following arthroscopic SCR using a dermal allograft, the early high failure rates of the graft raise concerns about the long-term outcomes of the procedure. Furthermore, the use of ultrasound alone to validate an intact graft should be used with caution, as failures can occur at the glenoid and can be missed without MRI correlation. Level of Evidence: Level IV, Therapeutic case series
\end{abstract}

$\mathbf{S}$ uperior capsular reconstruction (SCR) has been advocated as an effective technique for the management of massive irreparable rotator cuff tears, particularly in younger, active patients. ${ }^{1-4}$ There are few studies that demonstrate that arthroscopic SCR for

From Indiana University School of Medicine, Indianapolis, Indiana (B.L.B., A.M.B.); and Florida International University, Herbert Wertheim College of Medicine, Department of Medical and Population Health Sciences Research, Miami, Florida (M.M.), U.S.A.

The authors report the following potential conflicts of interest or sources of funding: Funding for obtaining advanced imaging for purposes of this study was granted by Arthrex. B.L.B. reports grants from Arthrex, during the conduct of the study; other from DJO Surgical, outside the submitted work. Full ICMJE author disclosure forms are available for this article online, as supplementary material.

Received October 8, 2019; accepted February 12, 2020.

Address correspondence to Brian L. Badman, M.D., 8607 E US 36, Avon, IN 46123.E-mail:bbadman@gmail.com

(C) 2020 THE AUTHORS. Published by Elsevier Inc. on behalf of the Arthroscopy Association of North America. This is an open access article under the CC BY-NC-ND license (http://creativecommons.org/licenses/by-nc-nd/4.0/). 2666-061X/191232

https://doi.org/10.1016/j.asmr.2020.02.006 irreparable rotator cuff tears improves stability and function of the shoulder joint. ${ }^{1,4,5}$ Despite the increasing popularity of the technique, only a relatively small number of patients in a few retrospective studies $^{6,7}$ and prospective studies ${ }^{5,8-10}$ exist regarding overall outcomes of the procedure.

Fascia lata autograft was the original graft that was described for the technique for arthroscopic SCR. ${ }^{1,5}$ Dermal allograft for SCR was later proposed as an alternative, as it reduces donor-site morbidity and overall surgical time. ${ }^{11}$ Dermal allograft remains the most popular graft used in arthroscopic SCR. Only a few available studies have examined the survivability of the graft in patients, particularly with magnetic resonance imaging (MRI) techniques. ${ }^{5,6,7-9}$ Little is known regarding SCR dermal allograft integrity in patients by ultrasound (US).

US evaluation can be useful in the postoperative evaluation of rotator cuff repairs. ${ }^{12-14}$ There are advantages of the use of US in the postoperative shoulder examination. US evaluation allows for a dynamic examination of the shoulder and can be reliably 
performed in the office. US is highly operator dependent, however. MRI evaluation of the shoulder, however, is a static examination and is more costly and time-consuming.

The purpose of this study was to evaluate the shortterm outcomes of 10 patients with irreparable massive rotator cuff tears treated with arthroscopic SCR using dermal allograft. We hypothesized that SCR with dermal allograft would lead to significant improvements in clinical and functional patient outcomes and that the majority of dermal allografts would remain intact by MRI and US evaluation.

\section{Methods}

Between 2016 and 2018, patients with symptomatic irreparable rotator cuff tears were prospectively enrolled for treatment with arthroscopic SCR with dermal allograft. Informed consent and investigational review board approval was achieved for the purposes of the study (Salus IRB). All patients were treated by a single fellowship-trained shoulder surgeon. Range of motion and functional outcome were measured by the treating surgeon according to American Shoulder and Elbow Surgeons (ASES) score, ${ }^{15}$ Simple Shoulder Test (SST), ${ }^{16,17}$ and visual analog scale (VAS) pain ${ }^{18}$ score were assessed preoperatively and at routine follow-up intervals. Inclusion criteria included all patients treated with arthroscopic SCR with dermal allograft. Exclusion criteria included patients revised to reverse shoulder arthroplasty during the study period. Minimum follow-up was 1 year. All patients were managed preoperatively with maximum conservative measures and then were referred for arthroscopic SCR after conservative measures failed. This included periodic use of steroid in all patients. No patient was offered surgery within 3 months of recent injection.

Preoperatively, plain radiographs and MRI were obtained in all patients to confirm the presence of massive irreparable rotator cuff tears without significant glenohumeral degenerative change. In-office US was used preoperatively in those patients that did not arrive to their initial clinic visit with MRI confirmation of a tear. From standard radiographs, acromial humeral distance (millimeters) was radiographically measured preoperatively and 12 months postoperatively on a standard Grashey shoulder view. Shoulders were graded according to the well-described Hamada classification. ${ }^{19,20}$ Preoperative MRI was used additionally to assess the presence of a tangent sign and the degree of fatty degeneration of rotator cuff musculature, according to the Goutallier classification. ${ }^{21,22}$ All classifications and grading were made by the treating surgeon. Postoperatively, US and MRI evaluation of the shoulder were obtained at a minimum of 1 year to assess graft integrity and to correlate clinical outcomes.
Our surgical technique for arthroscopic SCR is similar to previously described techniques. ${ }^{3,9}$ Before SCR, the following were performed in all cases: subscapularis evaluation and repair, if needed, biceps evaluation with tenotomy or tenodesis, subacromial decompression with acromioplasty, and remaining infraspinatus repair, if possible. For SCR, each case used acellular dermal allograft secured by 2 glenoid anchors and 4 greater tuberosity anchors in a double-row configuration. Convergence suture techniques were used on the posterior aspect of the repair.

Postoperatively, the rehab protocol in place was very similar to protocols used for large or massive rotator cuff repairs with and without dermal augmentation. All patients were typically immobilized in a sling for 6 weeks. Rehabilitation through passive motion occurred between 4 and 8 weeks postoperatively. Patients would undergo active-assisted motion between 8 and 10 weeks, and then patients would start active motion after 10 weeks postoperatively. After 10 weeks, patients were started on a graduated strengthening that focused on deltoid, periscapular, and the posterior rotator cuff musculature. Patients were allowed to progress as tolerated thereafter.

\section{Statistical Analysis}

Mann-Whitney $U$ tests and Fisher exact tests were used to compare patient characteristics between intact and failed grafts. Wilcoxon signed-rank tests were performed to compare preoperative and l-year postoperative outcomes. Data were analyzed using SPSS, version 25 (IBM Corp., Armonk, NY). All statistical tests were 2 -tailed. Significance was set at $P<.05$.

\section{Results}

Ten patients were prospectively enrolled into the study. Ten patients with a mean age of 58.6 years had a minimum follow-up of 1 year. Two patients $(20 \%)$ had a previous rotator cuff repair. No patients were revised to reverse shoulder arthroplasty during the study period. Three patients were retired; the 7 remaining patients returned to their job. No patient performed strenuous manual labor.

At 1 year, all patients had both US and MRI to assess graft integrity. US identified graft failure in 1 patient $(10 \%)$, whereas MRI identified graft failure in 7 patients $(70 \%)$. As a diagnostic tool in this setting, US had a sensitivity of $14 \%$, specificity of $100 \%$, positive predictive value of $100 \%$, negative predictive value of $33 \%$, and accuracy of $40 \%$. Of the 7 failures based on MRI, 4 failed at the level of the glenoid, 2 failed mid graft, and 1 failed at the humerus. Figure 1 provides examples of corresponding US and MRI images of patients with intact and failed grafts.

Table 1 lists the patient demographics as well as scores from VAS pain, SST, and ASES questionnaires in 
Fig 1. US and MRI of selected intact and failed grafts. US and MRI were performed in all patients at 1 year following superior capsular reconstruction with dermal allograft. Single coronal images of corresponding US and Tl-weighted MRI are shown from left to right in 4 separate patients. Structures in the US images are labeled as D (deltoid), G (graft), H (humerus). (A), US shows an intact graft, whereas MRI in the same patient shows graft failure mid-graft. (B), US shows an intact graft, whereas MRI in the same patient reveals graft failure at the glenoid. (C), US shows complete graft failure along with a confirmatory corresponding MRI. (D), images demonstrate an intact graft on both US and MRI in the same patient. (MRI, magnetic resonance imaging; US, ultrasound.)
A
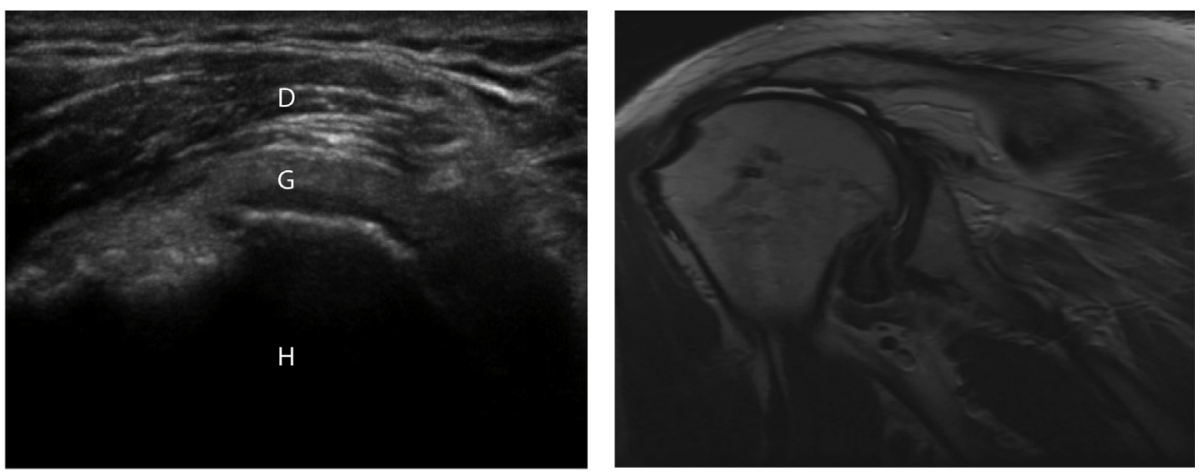

B
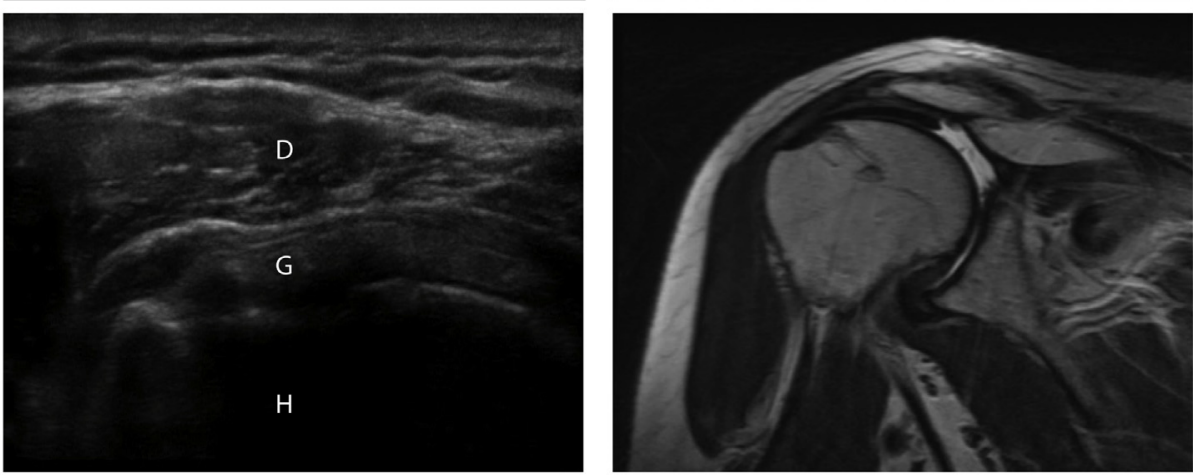

\section{C}

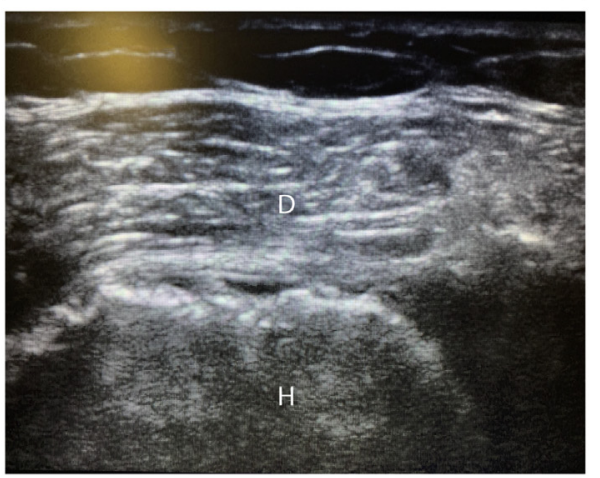

D

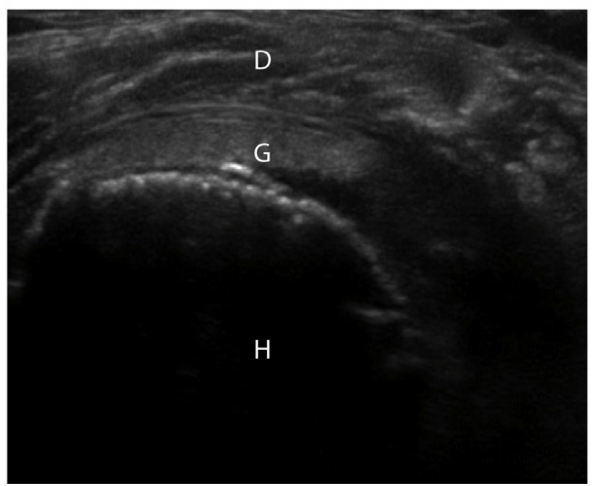

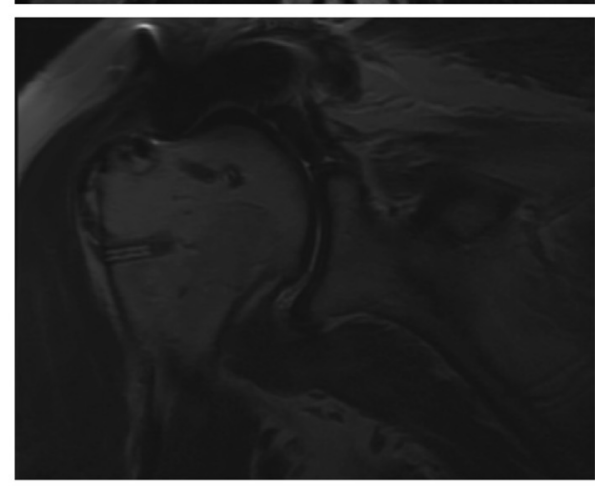

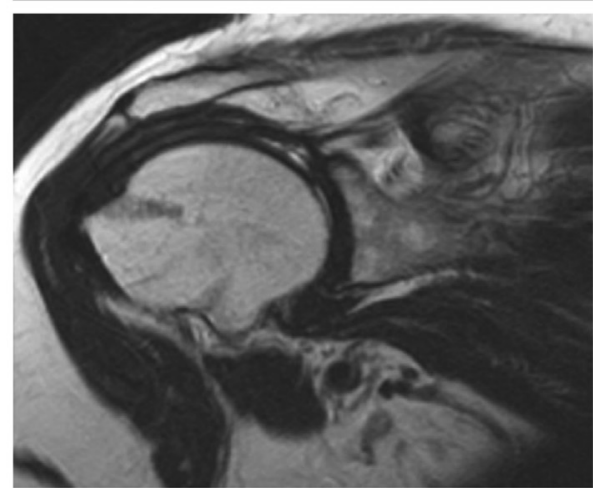

patients with intact grafts versus failed grafts. Age was greater in the intact group at 68.7 years compared with 54.3 years for the graft failure group $(P=.022)$. Sex was similar between groups $(P=.524)$. At 1 year postoperatively, there was no significant difference in VAS pain score $(P=.123)$, SST score $(P=.562)$, ASES score $(P=.909)$, abduction motion $(P=.673)$, or forward flexion motion $(P=.416)$ in patients with an intact graft versus those with a failed graft.

In all patients preoperatively to 1 year postoperatively, mean forward flexion improved from $141^{\circ}$ to $173^{\circ}(P=.018)$, mean abduction improved from 
Table 1. Comparison of Characteristics Between Patients With Intact and Failed Grafts Diagnosed by MRI at 1-Year Postoperatively

\begin{tabular}{|c|c|c|c|}
\hline Variable & $\begin{array}{l}\text { Intact Graft } \\
(\mathrm{n}=3), \\
\text { Mean } \pm \text { SD } \\
\text { or } \mathrm{n}(\%)\end{array}$ & $\begin{array}{l}\text { Failed Graft } \\
\quad(\mathrm{n}=7), \\
\text { Mean } \pm \text { SD } \\
\text { or } \mathrm{n}(\%)\end{array}$ & $P$ Value \\
\hline$\overline{\text { Age, } y}$ & $68.7 \pm 7.1$ & $54.3 \pm 4.2$ & .022 \\
\hline Male sex & $1(33.3)$ & $4(57.1)$ & .490 \\
\hline Previous surgery & $0(0)$ & $2(28.6)$ & .301 \\
\hline Operative side & & & $>0.999$ \\
\hline Right & $2(66.7)$ & $6(85.7)$ & \\
\hline Left & $1(33.3)$ & $1(14.3)$ & \\
\hline \multicolumn{4}{|l|}{ VAS pain } \\
\hline Preoperative & $5 \pm 0$ & $7 \pm 2.1$ & .271 \\
\hline 12 months postoperative & $0 \pm 0$ & $1.5 \pm 2.1$ & .123 \\
\hline \multicolumn{4}{|l|}{ SST } \\
\hline Preoperative & $3.3 \pm 1.5$ & $5.6 \pm 3.1$ & .353 \\
\hline First postoperative & $2.3 \pm 1.5$ & $2 \pm 1.4$ & .791 \\
\hline 6 weeks postoperative & $3 \pm 0$ & $4.3 \pm 1.8$ & .359 \\
\hline 3 months postoperative & $5 \pm 1$ & $8.1 \pm 2$ & .063 \\
\hline 6 months postoperative & $8.7 \pm 0.6$ & $9.1 \pm 2.9$ & .725 \\
\hline 12 months postoperative & $9.7 \pm 2.1$ & $10.3 \pm 2.1$ & .562 \\
\hline \multicolumn{4}{|l|}{ ASES Shoulder Score } \\
\hline Pre-op & $38.3 \pm 7.6$ & $44.7 \pm 11.6$ & .487 \\
\hline First postoperative & $48.3 \pm 17.2$ & $42 \pm 9.7$ & .563 \\
\hline 6 weeks postoperative & $55 \pm 2.8$ & $44.9 \pm 9.4$ & .140 \\
\hline 3 months postoperative & $69.7 \pm 13.7$ & $67.3 \pm 8.8$ & .646 \\
\hline 6 months postoperative & $88.7 \pm 1.2$ & $80 \pm 14.1$ & .435 \\
\hline 12 months postoperative & $90 \pm 8.9$ & $86.1 \pm 12.3$ & .909 \\
\hline \multicolumn{4}{|l|}{ Forward flexion ROM, } \\
\hline Preoperative & $120 \pm 87$ & $148 \pm 31$ & $>0.999$ \\
\hline 12 months postoperative, & $163 \pm 29$ & $176 \pm 10$ & .416 \\
\hline \multicolumn{4}{|l|}{ Abduction ROM } \\
\hline Preoperative & $130 \pm 86.6$ & $135 \pm 55.2$ & .663 \\
\hline 12 months postoperative & $150 \pm 52$ & $173 \pm 12.2$ & .673 \\
\hline
\end{tabular}

ASES, American Shoulder and Elbow Surgeons, MRI, magnetic resonance imaging; ROM, range of motion; $\mathrm{SD}$, standard deviation; SST, Simple Shoulder Test; VAS, visual analog scale.

$134^{\circ}$ to $166^{\circ}(P=.048)$, mean VAS pain decreased from 6.5 to $1.0(P=.004)$, SST improved from 4.9 to $10(P=$ $.005)$, and ASES improved from 43 to $87(P=.005)$ (Table 2). For those patients with an intact graft at 1 year, VAS pain decreased from 5 to $0(P=.083)$, SST improved from 3.3 to $9.7(P=.109)$, ASES improved from 38.3 to $90.0(P=.109)$, abduction improved from $130^{\circ}$ to $150^{\circ}(P=.317)$, and forward flexion improved from $120^{\circ}$ to $163^{\circ}(P=.18)$. For those patients with a failed graft at 1 year, VAS pain decreased from 7.0 to $1.5(P=.017)$, SST improved from 5.5 to $10(P=.017)$, ASES improved from 44.5 to $86(P=.018)$, abduction improved from $135^{\circ}$ to $173^{\circ}(P=.084)$, and forward flexion improved from $149^{\circ}$ to $176^{\circ}(P=.043)$.

Table 3 compares the preoperative imaging characteristics as well as the intraoperative findings in patients with intact and failed grafts based on their l-year postoperative MRI. There were no significant differences between the groups based on Goutallier classification of the supraspinatus $(P=.667)$, infraspinatus
$(P=1.0)$, or subscapularis $(P=1.0)$. The tangent sign was present preoperatively in all patients with an intact graft and $71 \%$ of patients with a failed graft $(P=1.0)$. Between the groups, all patients were grade 1 or 2 based on the Hamada classification $(P=1.0)$. Preoperatively, average acromial humeral distance was 7.4 $\mathrm{mm}$ and $6.6 \mathrm{~mm}$ for those with intact and failed grafts at 1 year, respectively $(P=.568)$. At 1 year, this measurement was $6.6 \mathrm{~mm}$ and $6.0 \mathrm{~mm}$ for those with intact and failed grafts, respectively $(P=.493)$. There were no differences in the intraoperative rotator cuff tear pattern or residual tendon length between those patients with an intact and failed graft at 1 year $(P=.65$ and $P=.569$, respectively). Performing repair of subscapularis or infraspinatus along with biceps management showed no difference between those with intact or failed grafts $(P=1.0, P=1.0$, and $P=1.0$, respectively).

\section{Discussion}

The findings within this study support the first portion of our hypothesis in that there was a significant improvement in clinical and functional outcomes at 1 year in all patients treated with arthroscopic SCR with dermal allograft. However, the second portion of our hypothesis was refuted in that 7 of 10 patients $(70 \%)$ demonstrated that the graft had failed at 1 year by MRI evaluation. Despite graft failures, range of motion and clinical and functional outcome scores improved and trended toward significance even in these patients. There were no significant preoperative radiographic or intraoperative differences between those patients with an intact or failed graft at ol year.

In our study, the improvement in forward flexion, ${ }^{5,9,10}$ VAS, ${ }^{6,8-10}$ and ASES $^{5,6,8-10}$ scores was similar to previously published studies. When looking at graft failure and re-tear, there are a wide range of reported values for failure. A case study by Zerr et al. ${ }^{23}$ in 2017 reported on an SCR with dermal allograft that failed at the glenoid based on MRI findings. Denard et al. ${ }^{9}$ performed MRI on a subset (20 of 59) of patients and determined that dermal allograft failure occurred in $55 \%$ ( 11 of 20 with the MRI) of those cases. In 2018, Lee and $\mathrm{Min}^{10}$ had performed MRI on all patients and reported a re-tear rate of $36.1 \%$ (13/36) for SCRs with dermal allograft between 6 and 12 months postoperatively. Contrary to our results, failures in both of these studies most often occurred on the humeral side rather than the glenoid side of the graft. ${ }^{9,10}$ Pennington et al. $^{6}$ reported a $4.5 \%$ clinical failure rate $(4 / 88)$ and $3.4 \%$ radiographic failure rate $(3 / 88)$ on MRI with dermal allograft, although only a small subset of those patients who were dissatisfied $(4 / 88)$ were imaged. So, of the 4 shoulders imaged in that study, $75 \%$ (3 of 4 ) showed failure radiographically. If we combine the results of all SCRs with dermal allograft in patients who 
Table 2. Preoperative and 1-Year Postoperative Comparisons Among Patients With Intact and Failed Grafts Diagnosed by MRI

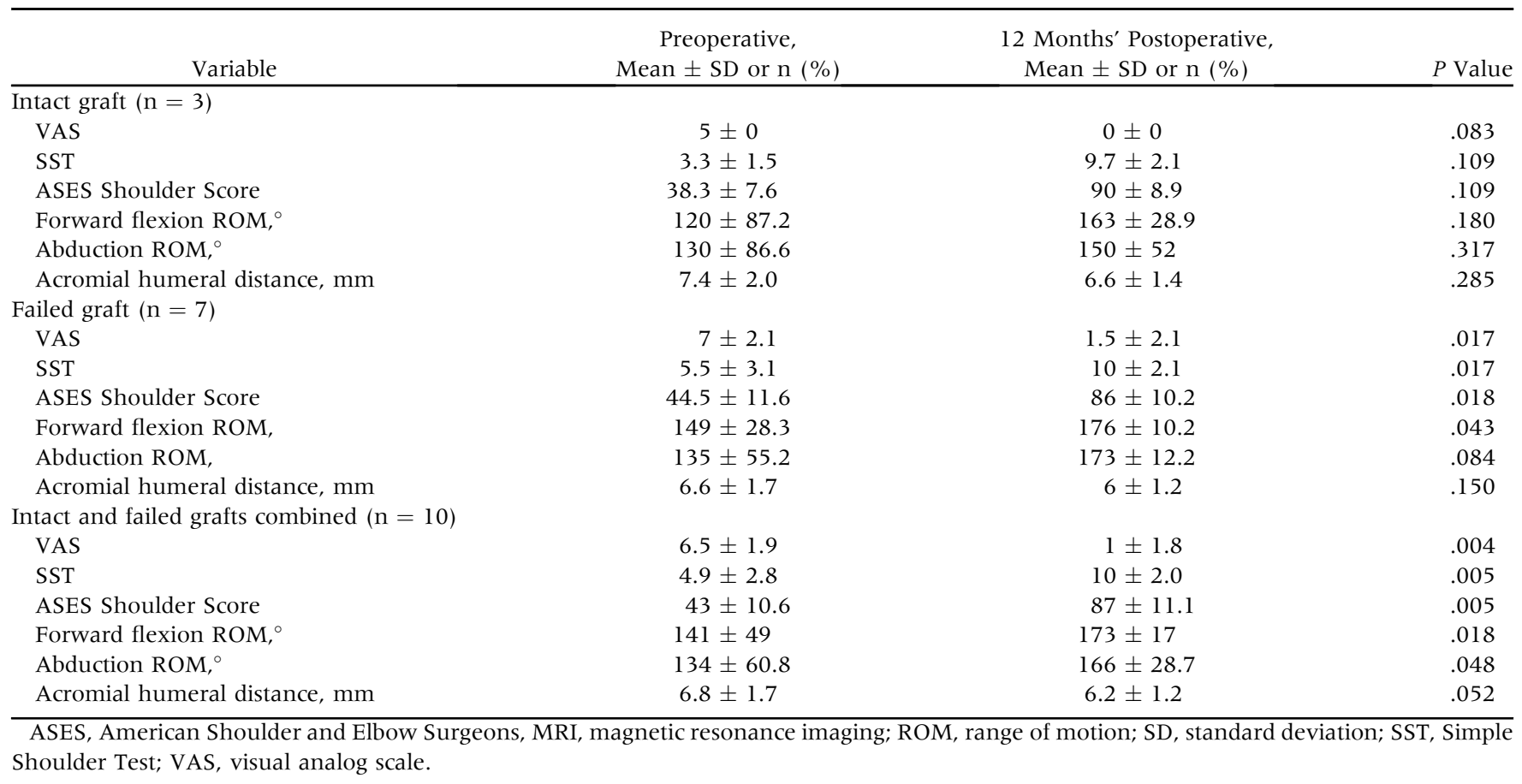

were imaged with MRI based on the aforementioned studies by Denard et al., Lee and Min, and Pennington et al., 45\% (27 of 60 ) of grafts failed on imaging, which is a fairly high rate of radiographic failure that is similar to the present study. Compared with the use of SCR with dermal allograft, the literature on SCR with fascia lata autografts is less abundant but may lead to some insight into radiographic graft failure. Mihata et al. ${ }^{24}$ showed that only $5 \%(5 / 100)$ of fascia lata autografts were torn on MRI by at least 2 years. The clinical and radiographic success of the use of autografts in this procedure may not be applicable to the use of allografts in this procedure. $^{25}$

US has been used reliably in the postoperative evaluation of rotator cuff repairs. ${ }^{12-14}$ Until recently, little was known regarding the utility of US in evaluation of healing after arthroscopic SCR. Hirahara et al. ${ }^{8}$ demonstrated that between 4 and 8 months after SCR, US can reveal pulsatile vessels within the allograft tissue, signifying incorporation and healing of the graft tissue. The present study used US to examine graft integrity in all patients and found that 1 of 10 patients had failure of the graft on US. However, when MRI was performed on all patients, an additional 6 graft failures were identified. Of the 7 graft failures, 4 total failures were at the glenoid, 2 failures were mid-graft, and 1 was at the level of the humerus. With poor sensitivity $(14 \%)$, negative predictive value $(33 \%)$, and overall accuracy $(40 \%)$, US should not be used alone to validate an intact graft, as failures are often missed on this imaging modality. Despite a radiologic graft failure rate of $70 \%$, all patients demonstrated clinical and functional improvement, which is consistent with data from available literature.

\section{Limitations}

There were several limitations to our study. First, follow-up data were only available for 1 year postoperatively. Long term, the data are unclear on the viability of SCR. Second, this study included a small sample size. This limited the ability to detect significant differences between patients with and without graft failure. A post-hoc power analysis revealed that greater than $80 \%$ power was achieved among both intact and failed graft groups in the preoperative to postoperative comparisons involving VAS pain, SST, and ASES; however, a minimum of $80 \%$ power was not achieved for range of motion or radiographic comparisons. Interestingly, when pooling all patients together, statistically significant preoperative to postoperative improvements were evident in almost all categories, despite limited power. Third, this study only used one fellowship-trained shoulder surgeon, who used dermal allograft for SCR procedures. There may be subtle variances in surgical technique between other surgeons that could lead to better radiographic outcomes, and the choice of graft may affect healing and graft incorporation.

\section{Conclusions}

Although clinical outcomes are statistically improved following arthroscopic SCR using a dermal allograft, the early high failure rates of the graft raise concerns about the long-term outcomes of the procedure. 
Table 3. Comparison of Preoperative Imaging and Intraoperative Characteristics Between Patients With Intact and Failed Grafts Diagnosed by MRI at 1 Year

\begin{tabular}{|c|c|c|c|}
\hline $\begin{array}{c}\text { Variable } \\
\end{array}$ & $\begin{array}{c}\text { Intact Graft }(\mathrm{n}=3) \\
\text { Mean } \pm \text { SD or } \mathrm{n}(\%)\end{array}$ & $\begin{array}{l}\text { Graft Failure }(\mathrm{n}=7) \text {, } \\
\text { Mean } \pm \text { SD or } \mathrm{n}(\%)\end{array}$ & $P$ Value \\
\hline Goutallier supraspinatus classification & & & .667 \\
\hline Stage 0 & $0(0)$ & $0(0)$ & \\
\hline Stage 2 & $0(0)$ & $1(14.3)$ & \\
\hline Stage 3 & $2(66.7)$ & $2(28.6)$ & \\
\hline Stage 4 & $1(33.3)$ & $4(57.1)$ & \\
\hline Stage 1 & $0(0)$ & $0(0)$ & \\
\hline Stage 2 & $0(0)$ & $0(0)$ & \\
\hline Stage 3 & $0(0)$ & $0(0)$ & \\
\hline Stage 4 & $0(0)$ & $0(0)$ & \\
\hline Goutallier infraspinatus classification & & & $>.999$ \\
\hline Stage 0 & $1(33.3)$ & $2(28.6)$ & \\
\hline Positive tangent sign & $3(100)$ & $5(71.4)$ & $>.999$ \\
\hline Hamada classification & & & 1.00 \\
\hline Grade 1 & $2(66.7)$ & $3(42.9)$ & \\
\hline Grade 2 & $1(33.3)$ & $4(57.1)$ & \\
\hline Grade 3 & $0(0)$ & $0(0)$ & \\
\hline Grade 4 & $0(0)$ & $0(0)$ & \\
\hline Grade 5 & $0(0)$ & $0(0)$ & \\
\hline \multicolumn{4}{|l|}{ Acromial humeral distance, $\mathrm{mm}$} \\
\hline Preoperative & $7.4 \pm 2.0$ & $6.6 \pm 1.7$ & .568 \\
\hline 12 months' postoperative & $6.6 \pm 1.4$ & $6.0 \pm 1.2$ & .493 \\
\hline Tear pattern & & & .650 \\
\hline Supraspinatus & $1(33.3)$ & $3(42.8)$ & \\
\hline Ruptured & $3(100)$ & $6(85.7)$ & \\
\hline
\end{tabular}

MRI, magnetic resonance imaging; SD, standard deviation.

Furthermore, the use of US alone to validate an intact graft should be used with caution, as failures can occur at the glenoid and can be missed without MRI correlation.

\section{References}

1. Mihata T, McGarry MH, Pirolo JM, Kinoshita M, Lee TQ. Superior capsule reconstruction to restore superior stability in irreparable rotator cuff tears: A biomechanical cadaveric study. Am J Sports Med 2012;40:2248-2255.

2. Adams CR, Demartino AM, Rego G, Denard PJ, Burkhart SS. The rotator cuff and the superior capsule: Why we need both. Arthroscopy 2016;32:2628-2637.

3. Burkhart SS, Denard PJ, Adams CR, Brady PC, Hartzler RU. Arthroscopic superior capsular reconstruction for massive irreparable rotator cuff repair. Arthroscopy Tech 2016;5:1407-1418.
4. Sochacki KR, McCulloch PC, Lintner DM, Harris JD. Superior capsular reconstruction for massive rotator cuff tear leads to significant improvement in range of motion and clinical outcomes: a systematic review. Arthroscopy 2019;35:1269-1277.

5. Mihata T, Lee TQ, Watanabe C, Fukunishi K, Ohue M, Tsujimura T, Kinoshita M. Clinical results of arthroscopic superior capsule reconstruction for irreparable rotator cuff tears. Arthroscopy 2013;29:459-470.

6. Pennington WT, Bartz BA, Pauli JM, Walker CE, Schmidt W. Arthroscopic superior capsular reconstruction with acellular dermal allograft for the treatment of massive irreparable rotator cuff tears: Short-term clinical outcomes and the radiographic parameter of superior capsular distance. Arthroscopy 2018;34:1764-1773.

7. Tokish JM, Momaya A, Roberson T. Superior capsular reconstruction with partial rotator cuff repair: A case report. JBJS Case Connect 2018:8el. 
8. Hirahara AM, Anderson WJ, Panero AJ. Superior capsular reconstruction: Clinical outcomes after minimum 2-year follow-up. Am J Orthop 2017;46:266-278.

9. Denard PJ, Brady PC, Adams CR, Tokish JM, Burkhart SS. Preliminary results of arthroscopic superior capsule reconstruction with dermal allograft. Arthroscopy 2018;34: 93-99.

10. Lee SJ, Min TK. Can inadequate acromiohumeral distance improvement and poor posterior remnant tissue be the predictive factors of re-tear? Preliminary outcomes of arthroscopic superior capsular reconstruction. Knee Surg Sports Traumatol Arthrosc 2018;26:2205-2213.

11. Hirahara AM, Adams CR. Arthroscopic superior capsular reconstruction for treatment of massive irreparable rotator cuff tears. Arthrosc Tech 2015;4:e637-e641.

12. Lee SJ, Williams D, Endo Y. The repaired rotator cuff: MRI and ultrasound evaluation. Curr Rev Musculoskelet Med 2018;11:92-101.

13. Pierce JL, Nacey NC, Jones S, et al. Postoperative shoulder imaging: Rotator cuff, labrum, and biceps tendon. Radiographics 2016;36:1648-1671.

14. Mack LA, Nyberg DA, Matsen FR 3rd, Kilcoyne RF, Harvey D. Sonography of the postoperative shoulder. AJR Am J Roentgenol 1988;150:108901093.

15. Richards RR, An KN, Bigliana LU, et al. A standardized method for the assessment of shoulder function. J Shoulder Elbow Surg 1994;3:34-352.

16. Godfrey J, Hamman R, Lowenstein S, Briggs K, Kocher M. Reliability, validity, and responsiveness of the simple shoulder test: Psychometric properties by age and injury type. J Shoulder Elbow Surg 2007;16:260-267.

17. Beaton D, Richards RR. Assessing the reliability and responsiveness of 5 shoulder questionnaires. J Shoulder Elbow Surg 1998;7:565-572.
18. Tashijian RZ, Deloach J, Porucznik CA, Powell AP. Minimal clinically important differences (MCID) and patient acceptable symptomatic state (PASS) for visual analog scales (VAS) measuring pain in patients treated for rotator cuff disease. J Shoulder Elbow Surg 2009;18:927-932.

19. Hamada K, Fukuda H, Mikasa M, Kobayashi Y. Roentgenographic findings in massive rotator cuff tears. A longterm observation. Clin Orthop Relat Res 1990:92-96.

20. Walch G, Edwards TB, Boulahia A, Nove-Josserand L, Neyton L, Szabo I. Arthroscopic tenotomy of the long head of the biceps in the treatment of rotator cuff tears: Clinical and radiographic results of 307 cases. J Shoulder Elbow Surg 2005; 14:238-246.

21. Goutallier D, Postel JM, Bernageau J, Lavau L, Voisin MC. Fatty muscle degeneration in cuff ruptures. Pre- and postoperative evaluation by CT scan. Clin Orthop Relat Res 1994:78-83.

22. Fuchs B, Weishaupt D, Zanetti M, Hodler J, Gerber C. Fatty degeneration of the muscles of the rotator cuff: Assessment by computed tomography versus magnetic resonance imaging. J Shoulder Elbow Surg 1999;8: 599-605.

23. Zerr J, McDermott JD, Beckmann NM, Fullick RK, Chhabra A. Case study: Failure of superior capsular reconstruction using dermal allograft. Skelet Radiol 2017;46:1585-1589.

24. Mihata T, Lee TQ, Fukunishi K, Itami Y, Fujisawa Y, et al. Return to sports and physical work after arthroscopic superior capsule reconstruction among patients with irreparable rotator cuff tears. Am J Sports Med 2018;46: 1077-1083.

25. Savoie FH. Editorial commentary: Superior capsular reconstruction: When a systematic review of a procedure can be misleading. Arthroscopy 2019;35:1278-1279. 